\title{
A NEW SPECIES OF THE GENUS CORONIPES (ACARI: HETEROSTIGMATA: SCUTACARIDAE) ASSOCIATED WITH RETICULITERMES SPERATUS KYUSHUENSIS (ISOPTERA: RHINOTERMITIDAE) FROM KOREA
}

\author{
Alexander A. Khaustov ${ }^{1 *}$, Jong-Ho Lee ${ }^{2}$, Seong-Jin Lee ${ }^{2}$ and Jin-Sik Park
}

\author{
${ }^{1}$ Tyumen State University, Tyumen, Russia \\ ${ }^{2}$ Animal and Plant Quarantine Agency (QIA), Anyang, South Korea \\ *corresponding author; e-mail: alex1973khaustov@gmail.com
}

\begin{abstract}
A new species of the genus Coronipes Mahunka, 1972 (Acari: Pygmephoroidea: Scutacaridae), C. sperati sp. n. is described from termites Reticulitermes speratus kyushuensis Morimoto, 1968 (Isoptera: Rhinotermitidae) from South Korea. The genus Coronipes is redefined. A key to Coronipes species is provided. An association of pygmephoroid mites with termites is discussed.
\end{abstract}

KEY WORDS: Acari, Pygmephoroidea, systematics, termites, association, phoresy.

DOI: $10.21684 / 0132-8077.2016 .24 .1 .17 .25$

\section{INTRODUCTION}

The superfamily Pygmephoroidea Cross, 1965 includes 4 families: Pygmephoridae Cross, 1965, Neopygmephoridae Cross, 1965, Microdispidae Cross, 1965 and Scutacaridae Oudemans, 1916 and more than 1200 species (Zhang et al. 2011). Probably all pygmephoroid mites are fungivorous (Khaustov 2008), but some species of the family Microdispidae might be parasitoids of insects (Kaliszewski et al. 1995). Many pygmephoroid mites are associated with various insects and utilize them for phoresy (Kaliszewski et al. 1995). Members of Pygmephoridae, the early derivative family of Pygmephoroidea, are usually phoretic on Coleoptera and Diptera (Rahiminejad et al. 2015), while Neopygmephoridae, Microdispidae and Scutacaridae, which form a monophyletic group of derived pygmephoroid mites, are mainly phoretic on Hymenoptera, especially on various ants (Ebermann and Moser 2008; Khaustov 2008, 2014a, b; Ebermann et al. 2013). Association of pygmephoroid mites with termites is poorly studied. Representatives of all four families of pygmephoroid mites have been recorded as associates of various termites (Silvestri 1918; Mahunka 1964, 1966, 1981; Cross 1965; Cross and Moser 1971; CostaLeonardo and Soares 1993; Kurosa 1994; Wang et al. 2002). More detailed information about association of pygmephoroid mites with termites is provided in discussion paragraph. The cosmopolitan family Scutacaridae is the largest in the superfamily Pygmephoroidea and includes 24 genera and more than 800 species (Zhang et al. 2011). The genus Coronipes Mahunka, 1972 currently includes three described species (Mahunka, 1966, 1972; Mahunka and Mahunka-Papp 1988), one of which, C. samsinaki (Mahunka, 1966) is described from termites Coptotermes formosanus Shiraki, 1909 from China (Mahunka 1966).

During a study of mites associated with termite Reticulitermes speratus kyushuensis Morimoto, 1968 in South Korea, a new species of the genus Coronipes was revealed. The main aim of this paper is to describe new species of Coronipes from Korea.

\section{MATERIAL AND METHODS}

Mites were collected from termites Reticulitermes speratus kyushuensis and mounted in Hoyer's medium. The terminology of idiosoma and legs follows that of Lindquist (1986); the nomenclature of subcapitular setae and the designation of cheliceral setae follow those of Grandjean (1944, 1947), respectively. The system of Pygmephoroidea follows that of Khaustov $(2004,2008)$. All measurements are given in micrometers $(\mu \mathrm{m})$. For leg chaetotaxy the number of solenidia is given in parentheses. The holotype and nine paratypes are deposited in the mite collection of the Tyumen State University Museum of Zoology, Tyumen, Russia, 13 paratypes are deposited in the QIA, Gimcheon, South Korea. Photographs were taken with the aid of digital camera Hitachi KP-HD20A and the compound microscope Axio Imager. A2, Carl Zeiss, Germany and Axio Cam MRc and the compound microsope Discovery V20, Carl Zeiss, Germany.

\section{SYSTEMATICS}

\section{Family Scutacaridae Oudemans, 1916}

Genus Coronipes Mahunka, 1972

Type species: Coronipes divergens Mahunka, 1972 , by original designation. 


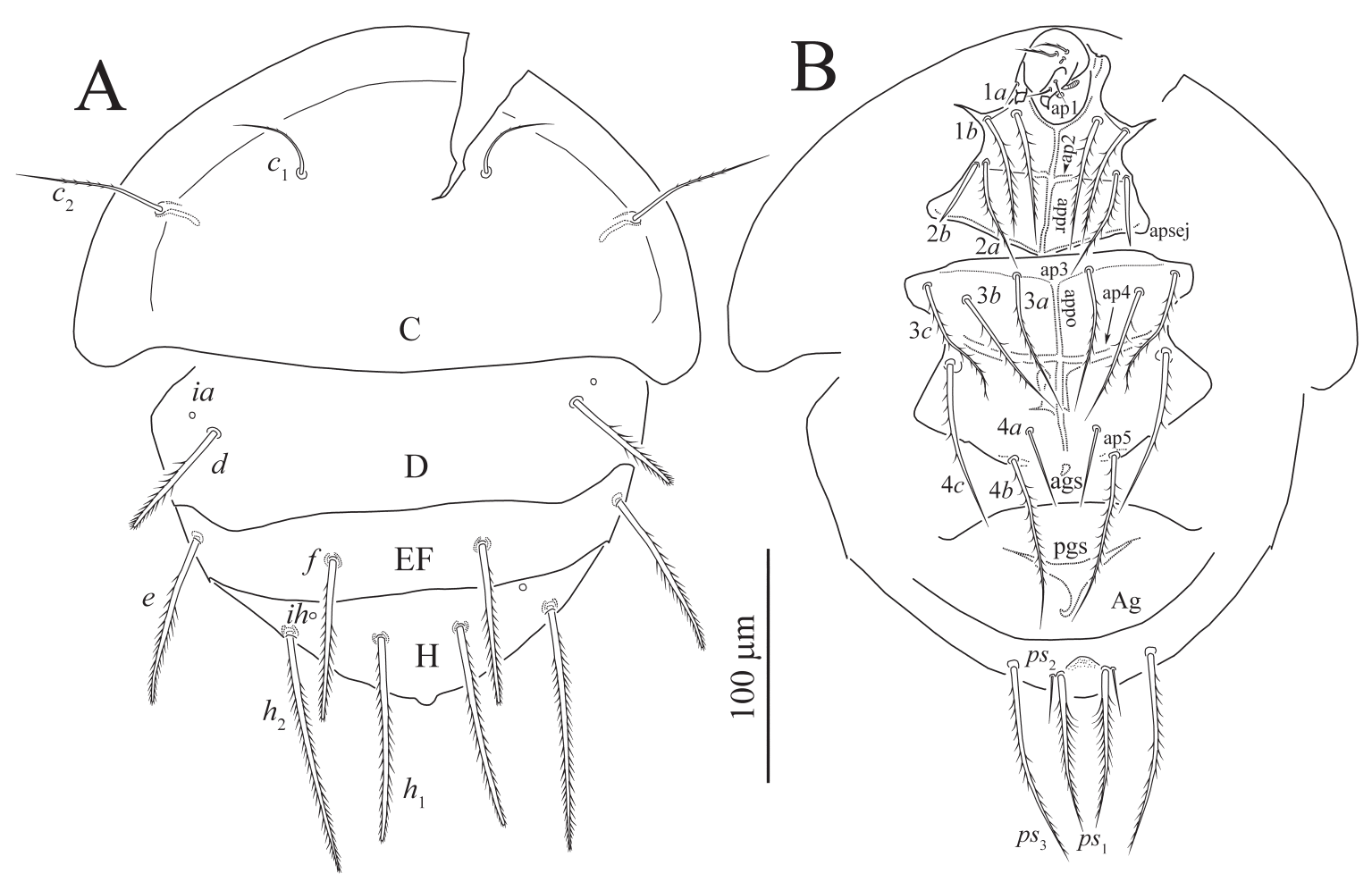

Fig 1. Coronipes speratii Khaustov, Lee, Lee et Park sp. n., female: A —idiosomal dorsum, B-idiosomal venter.

Diagnosis. Female. Body round. Prodorsum and gnathosoma completely covered by tergite $\mathrm{C}$. Gnathosomal capsule of similar length and width, dorsally with two pairs of cheliceral setae (cha and $c h b)$ and pair of dorsolateral postpalpal setae $(p p)$; palps prominent, with two pairs of dorsolateral setae $(\mathrm{dFe}, d \mathrm{Ge})$ and distinct tibial claw distally. Palps ventrally with mushroom-like accessory setigenous structure and well developed solenidion. Pharyngeal pumps grouped together; pharyngeal pump 2 distinctly longer than pharyngeal pumps 1 and 3. Prodorsum with two pairs of simple setae $\left(v_{2}\right.$ and $\left.s c_{2}\right)$, pair of clavate trichobothriae $\left(s c_{1}\right)$ and oval stigmata. Lateral propodosomal spine welldeveloped. Tergite $\mathrm{C}$ with two pairs of setae $\left(c_{1}\right.$, $\left.c_{2}\right)$; tergite $\mathrm{D}$ with one pair of setae $(d)$ and pair of round cupules $i a$; tergite $\mathrm{EF}$ with two pairs of setae $(e, f)$; tergite $\mathrm{H}$ with two pairs of setae $\left(h_{1}, h_{2}\right)$ and pair of round cupules $i h$; posterior margin of tergite $\mathrm{H}$ with short tongue-like process. Coxal fields I with two pairs of setae $(1 a, 1 b)$; coxal fields II with two pairs of setae $(2 a, 2 b)$; coxal fields III with three pairs of setae $(3 a, 3 b, 3 c)$; coxal fields IV with three pairs of setae $(4 a, 4 b, 4 c)$. Pseudanal segment with three pairs of setae $\left(p s_{1-3}\right)$. Apodemes 2 (ap2) short, joined with prosternal apodeme (appr); sejugal apodeme (apsej) well developed and joined with appr; secondary transverse apodeme (sta) present; apodemes 3 (ap3) well developed, diffuse, joined with poststernal apodeme (appo), apodemes 4 (ap4) well developed, long. Apodemes 5 (ap5) shorter, situated near the base of trochanter IV. Anterior genital sclerite (ags) short, bell-like; posterior genital sclerite (pgs) large, triangular. Setae $2 b$ smooth, slightly thickened. Leg I distinctly shorter and thinner than leg II. Tibiotarsus I tapering distally, with small claw situated on short pretarsus; eupathidia $t c$ " and $f t$ " situated on long pinnaculum; eupathidion $f t$ " situated in middle part of pinnaculum; seta $k$ long, pointed and smooth. Legs II, III with pair of large padded claws and flipper-like empodium. Setae $p l$ " on tarsi II and III distinctly thickened and dentate distally (Fig. 5B). Legs IV 5-segmented. Trochanter IV short and wide. Tibia and tarsus immovably connected. Pretarsus IV long and narrow, without claws; setae $p l$ " of tarsus IV absent. Leg chaetotaxy: leg I; tr $1\left(v^{\prime}\right)$, fe $3\left(d, l^{\prime}, v^{\prime \prime}\right)$, ge $4\left(l^{\prime}, l^{\prime}, v^{\prime}\right.$, $\left.v^{\prime \prime}\right)$, tita 16(4) (d, l', l', $v^{\prime}, v^{\prime \prime}, k,, t c^{\prime}, t c^{\prime \prime}, f t^{\prime}, f t^{\prime \prime}$, $p$ ", 'pl', $p l$ ', $p v ', p v$ ', $\left.s, \omega_{1}, \omega_{2}, \varphi_{1}, \varphi_{2}\right)$; leg II: tr 1 $\left(v^{\prime}\right)$, fe $3\left(d, l^{\prime}, v^{\prime \prime}\right)$, ge $3\left(l^{\prime}, l^{\prime \prime}, v^{\prime}\right)$, ti 4(1) $\left(d, l^{\prime}\right.$, $\left.v^{\prime}, v^{\prime \prime}, \varphi\right)$, ta 6(1) ( $\left.p l^{\prime \prime}, t c^{\prime}, t c^{\prime \prime}, u^{\prime}, p v^{\prime}, p v^{\prime \prime}, \omega\right)$; leg III: tr $1\left(v^{\prime}\right)$, fe $2\left(d, v^{\prime}\right)$, ge $2\left(l^{\prime}, v^{\prime}\right)$, ti 4(1) $\left(d, l^{\prime}\right.$, $\left.v^{\prime}, v^{\prime \prime}, \varphi\right)$, ta 6 ( $\left.p l^{\prime \prime}, t c^{\prime}, t c^{\prime \prime}, u^{\prime}, p v^{\prime}, p v^{\prime \prime}\right)$; leg IV: $\operatorname{tr} 1\left(v^{\prime}\right)$, fe $2\left(d, v^{\prime}\right)$, ge $1\left(v^{\prime}\right)$, ti 3(1) $\left(d, l^{\prime}, v^{\prime}, \varphi\right)$, ta 5 ( $\left.t c^{\prime}, t c^{\prime \prime}, u, p v^{\prime}, p v^{\prime \prime}\right)$. 

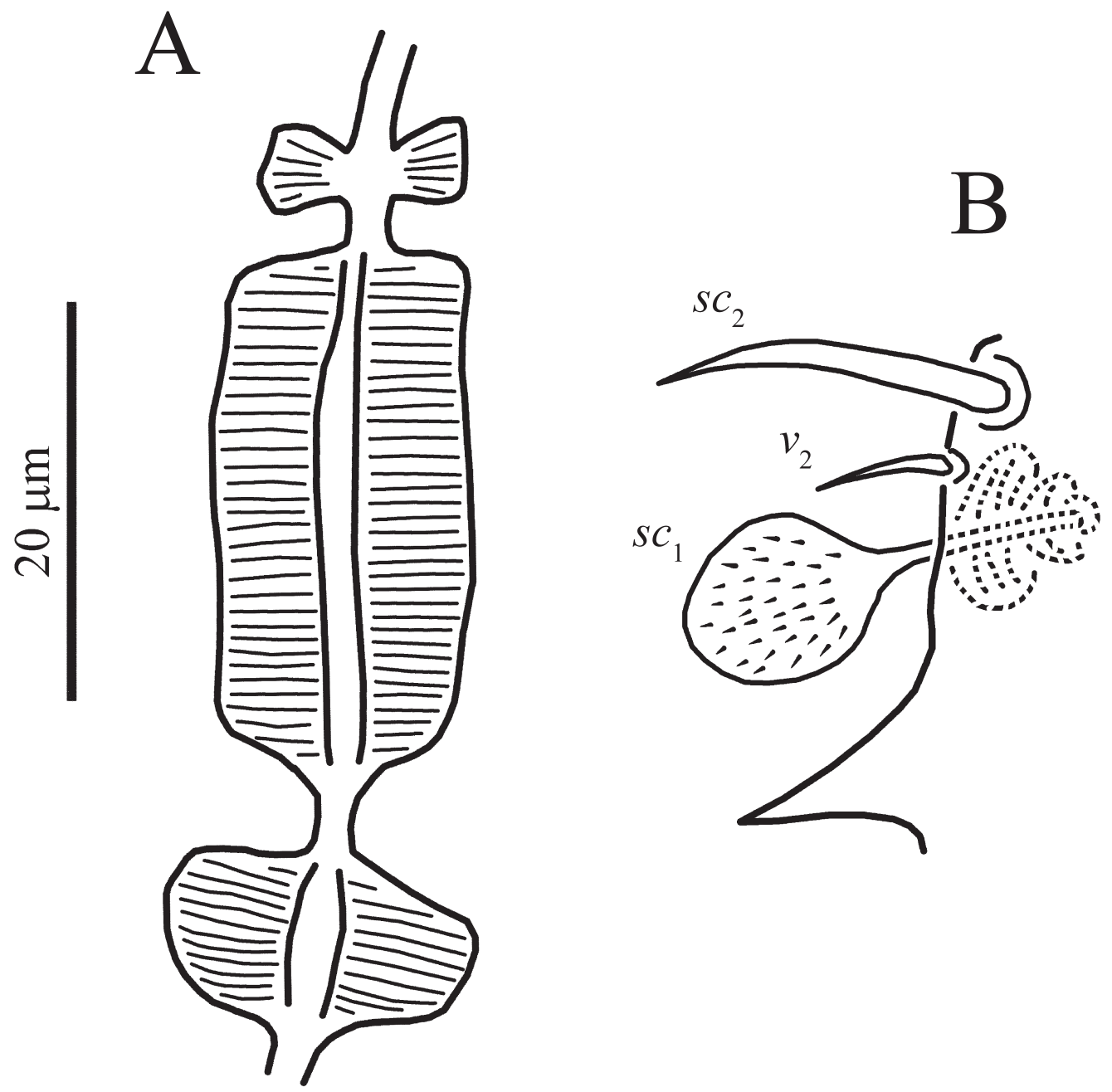

Fig. 2. Coronipes speratii Khaustov, Lee, Lee, Park sp. n., female: A — pharyngeal pumps, B—prodorsal setae.

Remarks. The genus Coronipes is very similar to Imparipes Berlese, 1903. It can be distinguished from Imparipes by dentate tip of setae $p l$ " on tarsi II and III (spine-like in Imparipes), absence of claws and setae $p l$ " on tarsus IV (usually present in Imparipes s.str.).

Species Included. The genus Coronipes includes three species, C. samsinaki (Mahunka, 1966), C. divergens Mahunka, 1972 and C. hauseri Mahunka and Mahunka-Papp, 1988.

Distribution and Habitat. Coronipes samsinaki is described from China (Mahunka 1966) and phoretic on termites Coptotermes formosanus Shiraki, 1909 (Isoptera: Rhinotermitidae); C. divergens described from New Guinea (Mahunka 1972) from litter accumulated at the base of sago palm; C. hauseri is described from Sabah (Eastern Malaysia) from forest litter (Mahunka and Mahunka-Papp 1988); C. sperati sp. n. phoretic on termites Reticulitermes speratus kyushuensis Morimoto, 1968 in South Korea.

\section{Coronipes sperati Khaustov, Lee, Lee et Park sp. $\mathbf{n}$.}

Figs. 1-6

Description. Female (Figs. 1-6). Length of idiosoma 290 (280-335), width 260 (255-300). Gnathosoma (Figs. 1B, 2A, 5A). Dorsal median apodeme present. Cheliceral setae subequal, sparsely barbed. Postpalpal setae ( $p p)$ claviform (Fig. 5A). Palpal solenidion very large, sausagelike. Setae $d G e$ and $d F e$ smooth, subequal. Pharyngeal pump as on figure 2A.

Idiosomal dorsum (Figs. 1A, 2B). Prodorsal setae smooth; $s c_{2}$ more than two times longer than $v_{2}$; trichobothria with numerous barbs (Fig. 2B). All hysterosomal tergites smooth. Setae $c_{1}$ and $c_{2}$ pointed, sparsely barbed, other dorsal setae bluntended and heavily barbed. Bases of setae $c_{2}$ associated with well-developed alveolar canal. Cupules $i a$ on tergite $\mathrm{D}$ and ih on tergite $\mathrm{H}$ large, round. Lengths of dorsal setae: $c_{1} 50$ (45-51), $c_{2} 62$ (60 72), d 53 (52-55), e 70 (69-76), f 68 (67-70), $h_{1}$ 


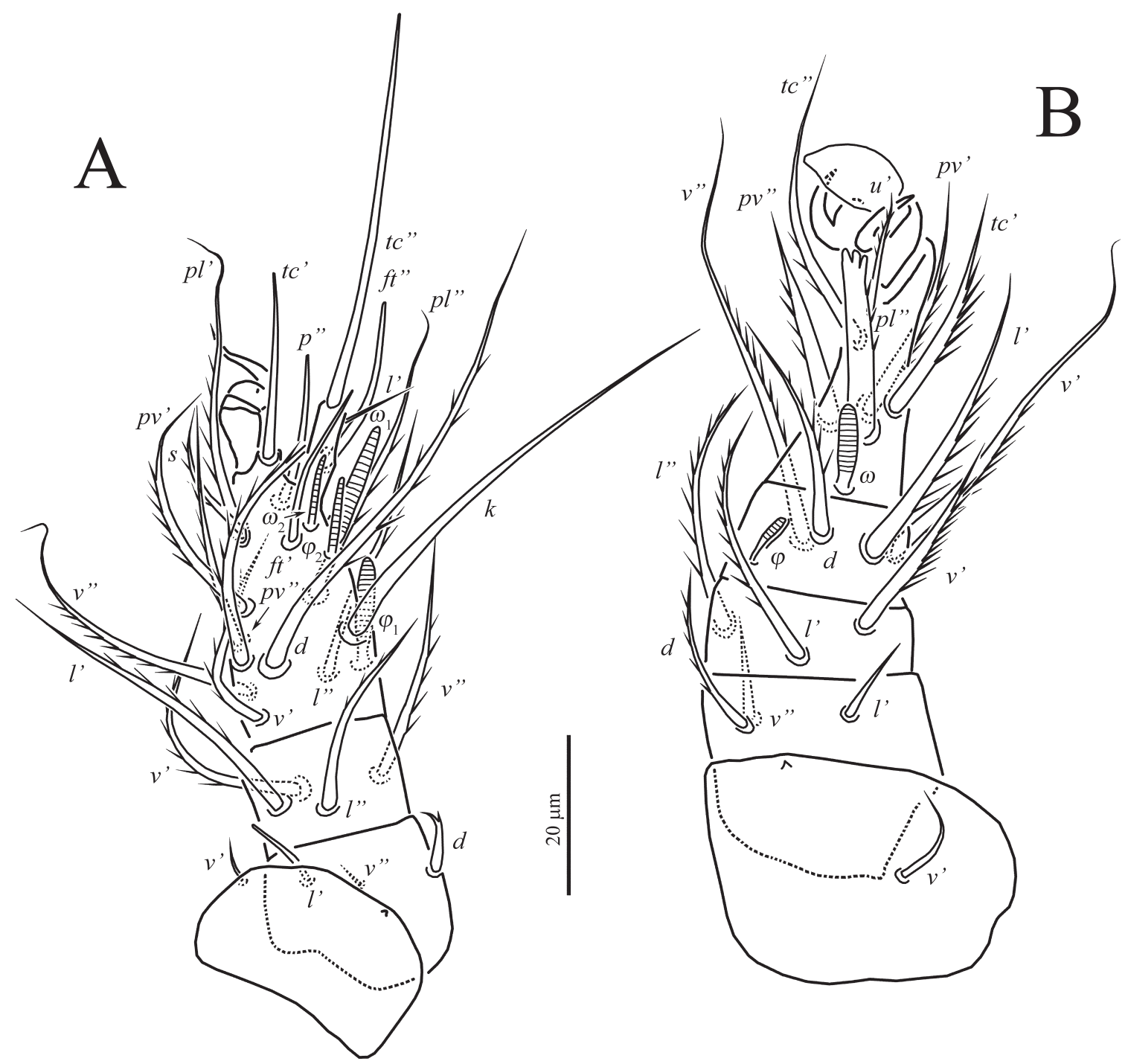

Fig. 3. Coronipes speratii Khaustov, Lee, Lee et Park sp. n., female: A-leg I, B-leg II.

88 (87-91), $h_{2} 105$ (105-110). Distances between setae: $c_{1}-c_{1} 71(70-77), d-d 160(160-170), e-f 64$ (62-65), f-f 67 (65-76), $h_{1}-h_{1} 35(34-40), h_{1}-h_{2} 40$ (39-42).

Idiosomal venter (Fig. 1B). All ventral plates smooth. Setae $4 a$ and $p s_{2}$ smooth, pointed. Setae $2 b$ smooth, slightly thickened, blunt-ended. Other ventral setae sparsely barbed and pointed. Posterior margin of posterior sternal plate distinctly concave. Posterior margin of aggenital plate rounded. Lengths of ventral setae: $1 a 57(55-60)$, $1 b 52$ (50-56), $2 a 54$ (52-59), $2 b 32$ (30-33), $3 a$ 65 (63-68), $3 b 65$ (63-68), $3 c 58$ (57-61), 4 a 36 (35-38), $4 b 72$ (70-77), 4c 66 (63-71), ps 68 (56-69), $p s_{2} 21$ (19-24), $p s_{3} 87$ (85-90). Legs (Figs. 3, 4, 5B). Leg I (Fig. 3A). Lengths of solenidia $\omega_{1}$ $16(15-16)>\omega_{2} 10(10-11)<\varphi_{1} 14(13-14)>\varphi_{2}$ 11 (11-12); $\omega_{2}$ and $\varphi_{2}$ baculiform, $\varphi_{1}$ and $\omega_{1}$ fingershaped. Setae $d F e$ slightly thickened and weakly barbed. Setae l'of femur smooth, blunt-ended; $v$ " of femur and $v$ ' of trochanter smooth, pointed; seta $k$ very long, smooth; other setae on leg segments (except eupathidia) sparsely barbed, pointed. Leg II (Fig. 3B). Solenidion $\omega 10$ (10-11), fingershaped, solenidion $\varphi 7$ (7-8) weakly clavate, situated in shallow depression. Setae $v$ 'of trochanter, $l$ ' and $v$ " of femur smooth, pointed; setae $p l$ " weakly barbed and with dentate tip (Fig. 5B); other setae on leg segments sparsely barbed, pointed. Leg III (Fig. 4A). Solenidion $\varphi 7$ (7-8) weakly clavate, situated in shallow depression. Setae $p l$ " as on tarsus II; other setae on leg segments sparsely barbed, pointed. Femur divided into basi- and telofemur. Leg IV (Fig. 4B). Empodium small, round. Solenidion $\varphi 8(8-9)$ weakly clavate, situated in shallow depression. Setae $d$ of femur and tibia blunt-ended, barbed; other setae on leg segments pointed, barbed. Femur divided into basi- 

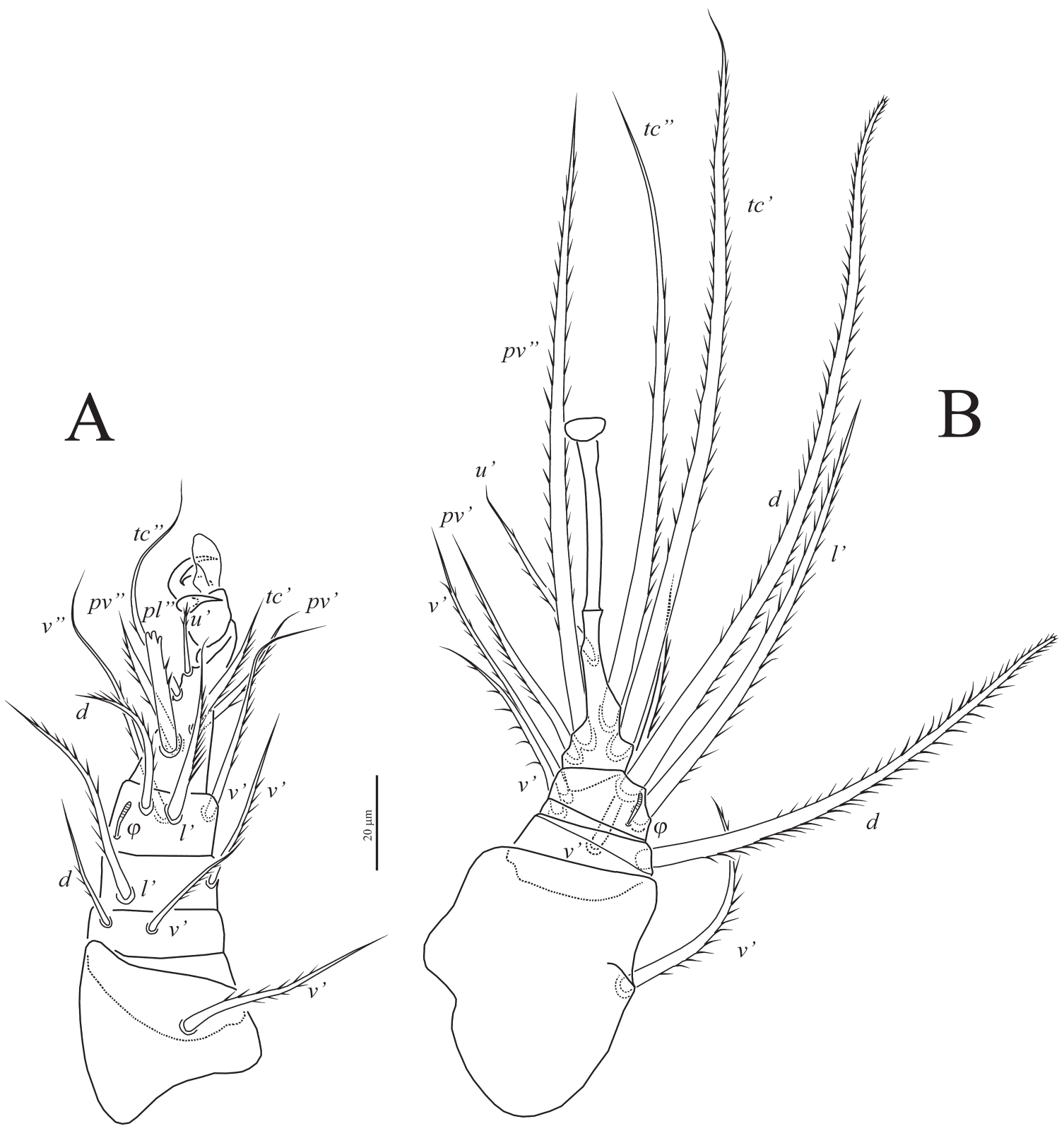

Fig. 4. Coronipes speratii Khaustov, Lee, Lee et Park sp. n., female: A—leg III, B-leg IV.

and telofemur. Length of tarsus IV 36 (35-37), length of pretarsus IV 36 (35-37).

Male and larva unknown.

Type material. Female holotype, slide \#YL181114, from termite colony taken at Mt. Oseong and reared in Honam Regional Office, QIA, South Korea, 18.11.2014, from 186-1, Yebang-ri, Seongsan-myeon, Gunsan city, Jeonbuk Prov., South Korea, 28.05.2014; paratypes: 9 females, same date and locality.

Etymology. The species name sperati derived from the species name of its phoretic host, Reticulitermes speratus.

Differential diagnosis. The new species is most similar to Coronipes samsinaki (Mahunka,
1966), but differs by setae $e$ slightly longer than $f$ (vs. $e$ distinctly shorter than $f$ in $C$. samsinaki) and by setae $p s_{2}$ distinctly shorter than half length of setae $p s_{1}$ (vs. $p s_{2}$ distinctly longer than half length of setae $p s_{1}$ in C. samsinaki). The new species differs from $C$. divergens Mahunka, 1972 by setae $e$ distinctly longer than $d$ (vs. setae $d$ and $e$ subequal in $C$. divergens). The new species differs from $C$. hauseri Mahunka and Mahunka-Papp, 1988 by setae $e$ only slightly shorter than $h_{2}$ (vs. $e$ about two times shorter than $h_{2}$ in C. hauseri).

\section{A key to females of the genus Coronipes}

1. Setae $d$ slightly longer or subequal to $e$..........2

- Setae $d$ distinctly shorter than $e$...................... 3 


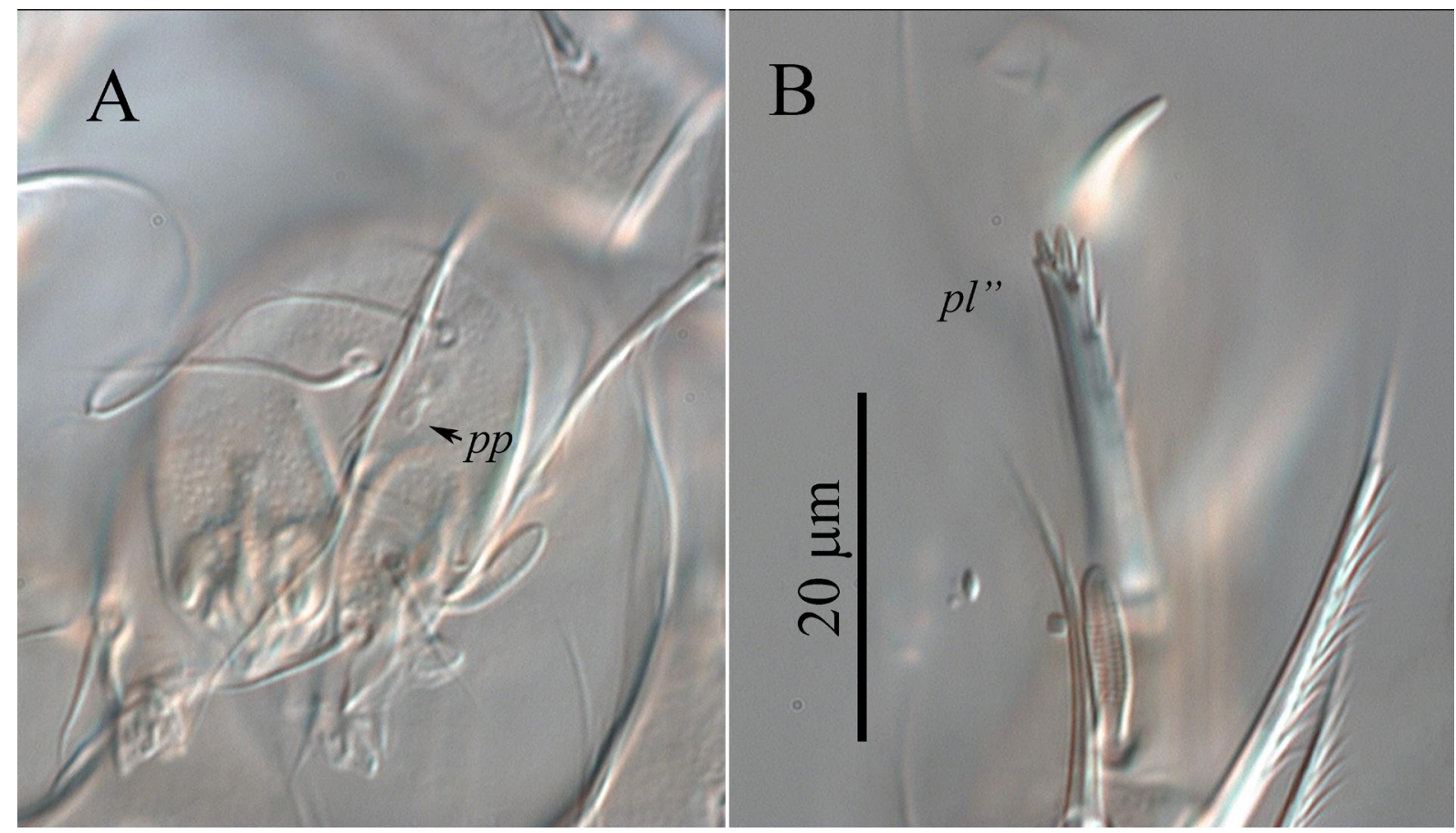

Fig. 5. Coronipes speratii Khaustov, Lee, Lee et Park sp. n., female, DIC micrographs: A—gnathosoma, B—-tarsus II in dorsal view.

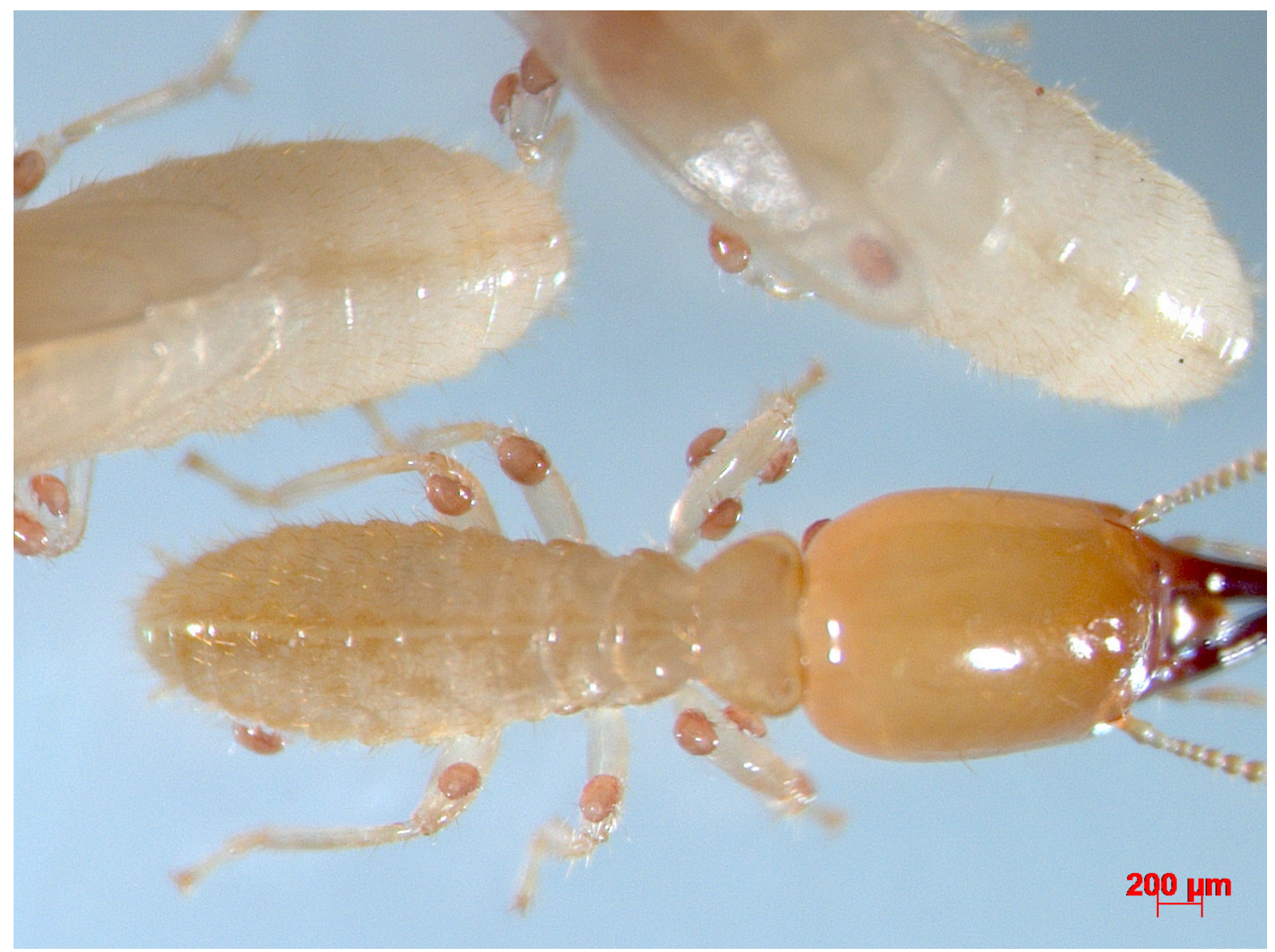

Fig. 6. Coronipes speratii Khaustov, Lee, Lee et Park sp. n., females phoretic on workers of Reticulitermes speratus kyushuensis. Stereo micrograph. 
2. Setae $p s_{2}$ distinctly shorter than half length of setae $p s_{1}$.................... divergens Mahunka, 1972 - Setae $p s_{2}$ distinctly longer than half length of setae $p s_{1}$............... C. samsinaki (Mahunka, 1966) 3. Setae $e$ only slightly shorter than $h_{2}$

C. sperati sp. $\mathrm{n}$.

- Setae $e$ about two times shorter than $h_{2} \ldots \ldots \ldots . . .$. ....C. hauseri Mahunka and Mahunka-Papp, 1988

\section{DISCUSSION}

Mites of the superfamily Pygmephoroidea associated with termites are poorly studied. All data available to the authors are summarized in Table 1. Silvestri (1918) described Imparipes termitophilus Silvestri, 1918 (Scutacaridae) phoretic on termites Pericapritermes appellans Silvestri, 1914 from Western Africa. Mahunka (1964) described Archidispus brevisetus Mahunka, 1964 on termites Odontotermes nolaensis and Imparipes angolensis Mahunka, 1964 (Scutacaridae) on Pseudacanthotermes spiniger (Sjostedt) from Angola. He also described Coronipes samsinaki (Mahunka, 1966) (Scutacaridae) from China (Mahunka 1966) on termites Coptotermes formosanus Shiraki, 1909. Delfinado and Baker (1976) described Imparipes adleri Delfinado and Baker 1976 from colony of Reticulitermes virginicus (Banks, 1907). Cross (1965) reported the finding of Parapygmephorus sp. (Neopygmephoridae) and Premicrodispus sp. (Microdispidae) on Reticulitermes flavipes (Kollar, 1837) (Rhinotermitidae) in Indiana, USA. Cross and Moser (1971) reported the discovery of Pygmephorellus sp. from Nasutitermes sp. in Texas, USA. Mahunka (1981) described a new genus and species, Luciaphorus hauseri Mahunka, 1981 (Pygmephoridae) from termite nest in St. Lucia, Antilles. Costa-Leonardo and Soares (1993) recorded phoresy of Pygmephorellus sp. and Luciaphorus hauseri on Heterotermes tenuis (Hagen, 1858) in Brazil. Kurosa (1994, 2002) described Nipponophorus ishikarai (Kurosa, 1994) and Mahunkania japonica Kurosa, 2002 and recorded Luciaphorus sp. in the nest of Reticulitermes speratus kyushuensis Morimoto, 1968.

Among nine known families of Isoptera, pygmephoroid mites are recorded as associates of two families: Termitidae and Rhinotermitidae (Table 1). Females of pygmephoroid mites are phoretic on termites, mainly on workers (Costa-Leonardo and Soares 1993). Pygmephorid mites are usually attached to legs of termites (Costa-Leonardo and Soares 1993). We recorded phoresy of Coronipes sperati $\mathrm{sp}$. n. on termite body and also found it in rearing medium, also mainly on legs of workers of Reticulitermes speratus kyushuensis (Fig. 6).

\section{REFERENCES}

Costa-Leonardo, A.M. and Soares, H.X. 1993. Occurrence of phoretic mites on the subterranean termite Heterotermes tenuis (Isoptera: Rhinotermitidae). Sociobiology, 23: 63-69.

Cross, E.A. 1965. The generic relationships of the family Pyemotidae (Acarina, Trombidiformes). The University of Kansas Science Bulletin, 45: 29215.

Cross, E.A. and Moser, J.C. 1971. Taxonomy and biology of some Pyemotidae (Acarina: Tarsonemoidea) inhabiting bark beetle galleries in North American conifers. Acarologia, 13(1): 47-64.

Delfinado, M.D. and Baker, E.W. 1976. New species of Scutacaridae (Acarina) associated with insects. Acarologia, 18(2): 264-301.

Grandjean, F. 1944. Observations sur les Acariens de la famille des Stigmaeidae. Archives des Sciences physiques et naturelles, 26: 103-131.

Grandjean, F. 1947. L'origine pileuse des mors et la chaetotaxie de la mandibule chez les Acariens actinochitineux. Comptes rendus des séances de l'Academie des Sciences, 224: 1251-1254.

Khaustov, A.A. 2004. [Mites of the family Neopygmephoridae Cross, 1965 stat. n. and their position in Heterostigmata]. In: Y.S. Balashov (Ed.). VIII Russian Acarological Conference, St.-Petersburg. Zoological Institute of RAS, St.-Petersburg, p. 137. [in Russian]

Khaustov, A.A. 2008. Mites of the Family Scutacaridae of Eastern Palaearctic. Akademperiodyka, Kiev, $291 \mathrm{pp}$.

Kurosa, K. 1994. A new genus and species of Pygmephoridae (Acari: Heterostigmata) associated with Reticulitermes spelatus kyushuensis (Isoptera: Rhinotermitidae) in Japan. Transactions of the Shikoku Entomological Society, 20 (3-4): 207214.

Kurosa, K. 2002. A new species of the genus $M a-$ hunkania (Acari: Heterostigmata: Pygmephoridae) from Japan. Journal of the Acarological Society of Japan, 11(2): 91-99.

Lindquist, E.E. 1986. The world genera of Tarsonemidae (Acari: Heterostigmata): a morphological, phylogenetic, and systematic revision, with a reclassification of family-group taxa in the Heterostigmata. Memoirs of Entomological Society of Canada, 136: 1-517. 
A. A. Khaustov, J.-H. Lee, S.-J. Lee and J.-S. Park

Mahunka, S. 1964. Neue Scutacariden aus Angola (Acari: Tarsonemini). Museo do undo, Subsidios para o estudo da biologia na lunda, 27: 117137.

Mahunka, S. 1966. Zwei neue Arten aus der Gruppe Tarsonemini aus China (Acari). Věstnik Československé Společnosti Zoologické, 30(4): 315-318.

Mahunka, S. 1972. The first survey of the Tarsonemid (Acari) fauna of New Guinea. I. Acta Zoologica Academiae Scientiarum Hungaricae, 18(1-2): 41-92.

Mahunka, S. 1981. Milben (Acari) aus St. Lucia (Antillen) I. Tarsonemina, Anoetidae. Acta Zoologica Academiae Scientiarum Hungaricae, 27 (3-4): 323-353.
Mahunka, S. and Mahunka-Papp, L. 1988. Neue und interessante Milben aus dem Genfer Museum LIX. Hauseripes hungarorum gen. n., sp. n. and some other new Tarsonemina (Acari) from Sabah (East Malaysia). Revue suisse de Zoologie, 95(2): 581-594.

Silvestri, F. 1918. Contribuzione alla conoscenza dei Termitidi e Termitofili dell' Africa occidentale. II. Termitofili. (1). Parte prima. Bollettino del Laboratorio di Zoologia General e Agraria, Portici, 12: 287-346.

Wang, C., Powell, J.E. and OConnor, B.M. 2002. Mites and nematodes associated with three subterranean termite species (Isoptera: Rhinotermitidae). Florida Entomologist, 85(3): 499-506.

Table 1. Known associations of mites with termites according to literature data

\begin{tabular}{|c|c|c|c|c|c|}
\hline \multirow{2}{*}{ Mite family } & \multirow{2}{*}{ Mite species } & \multicolumn{2}{|c|}{ Host termite } & \multirow{2}{*}{ Locality } & \multirow{2}{*}{ Reference } \\
\hline & & Termite family & Termite species & & \\
\hline \multirow{7}{*}{ Scutacaridae } & $\begin{array}{l}\text { Imparipes termitophilus } \\
\text { Silvestri, } 1918\end{array}$ & \multirow{3}{*}{ Termitidae } & $\begin{array}{l}\text { Pericapritermes } \\
\text { appellans Silvestri, } \\
1914\end{array}$ & $\begin{array}{l}\text { Western } \\
\text { Africa }\end{array}$ & $\begin{array}{l}\text { Silvestri } \\
1918\end{array}$ \\
\hline & $\begin{array}{l}\text { Archidispus brevisetus } \\
\text { Mahunka, } 1964\end{array}$ & & $\begin{array}{l}\text { Odontotermes } \\
\text { nolaensis Sjostedt, } \\
1924\end{array}$ & Angola & $\begin{array}{l}\text { Mahunka } \\
1964\end{array}$ \\
\hline & $\begin{array}{l}\text { Imparipes angolensis } \\
\text { Mahunka, } 1964\end{array}$ & & $\begin{array}{l}\text { Pseudacanthotermes } \\
\text { spiniger (Sjostedt, } \\
\text { 1900) }\end{array}$ & Angola & $\begin{array}{l}\text { Mahunka } \\
1964\end{array}$ \\
\hline & $\begin{array}{l}\text { Coronipes samsinaki } \\
\text { (Mahunka, 1966) }\end{array}$ & \multirow{6}{*}{ Rhinotermitidae } & $\begin{array}{l}\text { Coptotermes } \\
\text { formosanus Shi- } \\
\text { raki, } 1909\end{array}$ & China & $\begin{array}{l}\text { Mahunka } \\
1966\end{array}$ \\
\hline & $\begin{array}{l}\text { Coronipes sperati Khaustov, } \\
\text { Lee, Lee et Park sp. n. }\end{array}$ & & $\begin{array}{l}\text { Reticulitermes } \\
\text { speratus kyushuen- } \\
\text { sis Morimoto, } 1968\end{array}$ & $\begin{array}{l}\text { South } \\
\text { Korea }\end{array}$ & Present data \\
\hline & $\begin{array}{l}\text { Imparipes adleri Delfinado } \\
\text { et Baker, } 1976\end{array}$ & & $\begin{array}{l}\text { Reticulitermes } \\
\text { virginicus (Banks, } \\
\text { 1907) }\end{array}$ & USA & $\begin{array}{l}\text { Delfinado } \\
\text { and Baker } \\
1976\end{array}$ \\
\hline & Unknown genus and species & & $\begin{array}{l}\text { Reticulitermes } \\
\text { flavipes (Kollar, } \\
\text { 1837), Coptotermes } \\
\text { formosanus Shi- } \\
\text { raki, } 1909\end{array}$ & China & $\begin{array}{l}\text { Wang et al. } \\
2002\end{array}$ \\
\hline \multirow{2}{*}{ Neopygmephoridae } & Parapygmephorus sp. & & \begin{tabular}{|l} 
Reticulitermes \\
flavipes (Kollar, \\
1837)
\end{tabular} & USA & Cross 1965 \\
\hline & $\begin{array}{l}\text { Nipponophorus ishikarai } \\
\text { (Kurosa, 1994) }\end{array}$ & & $\begin{array}{l}\text { Reticulitermes } \\
\text { speratus kyushuen- } \\
\text { sis Morimoto, } 1968\end{array}$ & Japan & $\begin{array}{l}\text { Kurosa } \\
1994\end{array}$ \\
\hline
\end{tabular}


A new Coronipes (Acari: Scutacaridae) from Korea

\begin{tabular}{|c|c|c|c|c|c|}
\hline \multirow{3}{*}{ Microdispidae } & Premicrodispus sp. & \multirow{7}{*}{ Rhinotermitidae } & \begin{tabular}{|l} 
Reticulitermes \\
flavipes \\
(Kollar, 1837)
\end{tabular} & USA & Cross 1965 \\
\hline & Unknown genus & & $\begin{array}{l}\text { Reticulitermes } \\
\text { flavipes } \\
\text { (Kollar, 1837) }\end{array}$ & USA & Wang et al. 2002 \\
\hline & Near Unguidispus & & $\begin{array}{l}\text { Reticulitermes } \\
\text { flavipes } \\
\text { (Kollar, 1837) }\end{array}$ & USA & Wang et al. 2002 \\
\hline \multirow{5}{*}{ Pygmephoridae } & $\begin{array}{l}\text { Luciaphorus } \\
\text { hauseri Mahunka, } \\
1981\end{array}$ & & $\begin{array}{l}\text { Termite nest, } \\
\text { Heterotermes tenuis } \\
\text { (Hagen, 1858) }\end{array}$ & $\begin{array}{l}\text { St. Lucia, } \\
\text { Antilles, } \\
\text { Brazil }\end{array}$ & $\begin{array}{l}\text { Mahunka 1981; } \\
\text { Costa-Leonardo } \\
\text { and Soares } 1993\end{array}$ \\
\hline & Luciaphorus sp. & & $\begin{array}{l}\text { Reticulitermes } \\
\text { speratus kyushuensis } \\
\text { Morimoto, } 1968\end{array}$ & Japan & Kurosa 1994 \\
\hline & $\begin{array}{l}\text { Mahunkania } \\
\text { japonica Kurosa, } \\
2002\end{array}$ & & $\begin{array}{l}\text { Reticulitermes } \\
\text { speratus kyushuensis } \\
\text { Morimoto, } 1968\end{array}$ & Japan & Kurosa 2002 \\
\hline & Pygmephorellus sp. & & $\begin{array}{l}\text { Heterotermes tenuis } \\
\text { (Hagen, 1858) }\end{array}$ & Brazil & $\begin{array}{l}\text { Costa-Leonardo } \\
\text { and Soares } 1993\end{array}$ \\
\hline & Pygmephorellus sp. & Termitidae & Nasutitermes sp. & USA & $\begin{array}{l}\text { Cross and Moser } \\
1971\end{array}$ \\
\hline
\end{tabular}

